\title{
Cosmological Constraints from Cosmic Shear and CMB
}

\author{
I. Tereno \\ Institut d'Astrophysique de Paris, 98 bis, boulevard Arago, F-75014 Paris, France \\ Departamento de Física, Universidade de Lisboa, 1749-016 Lisboa, Portugal
}

\begin{abstract}
We analyse the sensitivity of cosmic shear 2-point statistics to the cosmological parameters, by using Monte Carlo Markov Chain (MCMC) methods. In particular, we investigate degeneracies among parameters and compare them to the CMB ones. Specializing on a Canada France Hawaii Telescope Legacy Survey (CFHTLS) type lensing survey and WMAP-1 year and CBI CMB data, we find cosmic shear information improves CMB cosmological parameters constraints by a factor of $1.5-2.5$.
\end{abstract}

\section{Introduction}

Propagation of galaxy light beams across large scale mass inhomogeneities produces distorted and magnified galaxy images (for reviews see Bartelmann \& Schneider (2001), Van Waerbeke \& Mellier (2003), see also ScHNEIDER contribution to these proceedings).

The exploration of these weak gravitational distortions (the cosmic shear) over fields of view as large as the CFHTLS-Wide has an enormous potential for cosmology. Past experiences based on first generation cosmic shear surveys (see for example a review in VAN WAERBEKE contribution to this volume), have demonstrated they can constrain the dark matter properties $\left(\sigma_{8}, \Omega_{m}\right.$ and the shape of the dark matter power spectrum) from a careful investigation of the ellipticity induced by weak gravitational shear on distant galaxies. Second generation cosmic shear surveys, being more sensitive to the cosmological parameters, will allow not only to ameliorate these constraints, but also to take into account a broader range of cosmological parameters and consequentely to more thoroughly investigate different cosmological models, like the inclusion of dark energy evolution (Benabed \& van Waerbeke (2003)) or of alternative primordial power spectra (Ishak et al. (2004)).

The full scientific outcome of cosmic shear data will only be complete if all information contained in the cosmic shear signal is used, including higher order statistics (Takada \& Jain (2004), see also KILBINGER, these proceedings) and 3-dimensional information (Bacon et al. (2004), see also KING, these proceedings) and if a joint analysis with other data sets is done (Contaldi et al. (2003)).

The focus of this talk is on this last issue : our goal is to map the parameter space that describes cosmological models in order to extract series of parameter combinations that minimise intersections of $\mathrm{CMB}$ and cosmic shear degeneracy tracks. For the complete details of our analysis refer to Tereno et al. (2004), of which this talk is an abridged version.

\section{Mapping the parameters' space}

In order to map the parameters' space, we need essentially to define a cosmic shear or CMB fiducial model, to put error bars on it and then assign a likelihood to each 
cosmological model in the parameters' space with respect to these cosmic shear or CMB data.

\subsection{Fiducial model}

We parameterize a cosmological model with a set of 13 parameters, $\left(\omega_{b}, \omega_{c}, \Omega_{v}, f_{\nu}, h ; \sigma_{8}, n_{s}, \alpha_{s}, r, n_{t} ; \tau ; w ; z_{s}\right)$, whose fiducial values are shown in Table 1.

\begin{tabular}{lllllll}
\hline$\omega_{b}=0.022$ & $\omega_{c}=0.114$ & $\Omega_{v}=0.73$ & $f_{\nu}=0$ & $\Omega_{k}=0$ & $h=0.71$ & \\
$n_{s}=0.93$ & $\alpha_{s}=-0.04$ & $r=0$ & $w=-1$ & $\tau=0.17$ & $\sigma_{8}=0.9$ & $z_{s}=0.8$
\end{tabular}

Table 1. Fiducial cosmological model.

The primordial scalar power spectrum,

$$
P_{i}(k)=A_{s}\left(\frac{k}{k_{0}}\right)^{n_{s}(k)-1},
$$

is allowed to have a scale dependent index, which is Taylor expanded around $k_{0}=0.05 h \mathrm{Mpc}^{-1}: n_{s}(k)=n_{s}+1 / 2 \alpha_{s} \ln \left(k / k_{0}\right)$.

From the primordial power spectrum and for a given matter/energy budget, consisting of baryons $\left(\omega_{b}\right)$, cold dark matter $\left(\omega_{c}\right)$, neutrinos $\left(f_{\nu}\right)$ and a cosmological constant $\left(\Omega_{v} ; w=-1\right)$, the liner matter power spectrum, $P_{\text {lin }}(k, z)=\left[D_{1}^{(+)}(z)\right]^{2} P_{\text {lin }}(k)$, is computed for an epoch $z$, using CAMB (Lewis et al. (2000)). The linear matter power spectrum is then renormalized with $\sigma_{8}$ and a non-linear power spectrum is evaluated from the linear one using the HALOFIT prescription of Smith et al. (2003).

The (dark) matter large scale distribution represented by this power spectrum is responsible for the cosmic shear on the source galaxies images, assuming other causes for shear correlations, like intrinsic alignments, are irrelevant. The shear correlations are then encoded in the power spectrum of the mass of the 2-dimensional projected gravitational potential of the lenses: the power spectrum of the convergence $\kappa$. This is computed from the contribution of all the lenses (the dark matter fluctuations) along the line of sight, by integrating the power spectrum along the radial coordinate $(w)$ between observer and source, multiplied by a lensing efficiency factor which depends on the angular diameter distances of the system source-lens-observer :

$$
P_{\kappa}(s)=\frac{9}{4}\left(\frac{H_{0}}{c}\right)^{4} \Omega_{m}^{2} \int_{0}^{w_{s}} d w\left(\frac{f_{K}\left(w_{s}-w\right)}{a(w) f_{K}\left(w_{s}\right)}\right)^{2} P\left(\frac{s}{f_{K}(w)} ; w\right) .
$$

Notice the source galaxies are all at the same redshift $z_{s}\left(w_{s}\right)$ and $s$ is the angular scale, $k=s / f_{K}(w) H_{0} / c[h / \mathrm{Mpc}]$.

One of the 2-point functions that is usually evaluated from shear measurements is the variance of the shear in a top-hat filter of radius $\theta$. This quantity relates to the power spectrum of the convergence through,

$$
\left\langle\gamma^{2}(\theta)\right\rangle=\frac{2}{\pi} \int_{0}^{\infty} \mathrm{d} s s P_{\kappa}(s)\left[\frac{J_{1}(s \theta)}{s \theta}\right]^{2} .
$$

We evaluated Eq. (2.3) for the fiducial cosmological model, obtaining thus a fiducial variance of the shear. This was done for 20 scale points, between $0.6^{\prime}$ and $120^{\prime}$. This range allows to probe both the non-linear and the linear regimes, the transition being at around $10^{\prime}$ or $k=0.3 h / \mathrm{Mpc}$. 


\subsection{Error bars}

Having obtained a reference shear variance, we must assign error bars to those 20 points. In order to do this we consider the estimator of the shear variance from observed, PSF corrected, galaxies ellipticities and compute its variance-covariance matrix, following Schneider et al. (2002). The result, valid for a Gaussian shear field, depends on the size of the field, on the density of galaxies and on their intrinsic ellipticity dispersion, as well as on the fiducial shear correlation functions. For these survey specifications we use the predicted features of the CFHTLS-Wide Legacy Survey $\dagger$, which are, $\left(A=170 \mathrm{deg}^{2} ; n_{g}=20 \operatorname{arcmin}^{-2} ; \sigma_{\epsilon}=0.4\right)$.

\subsection{Likelihood evaluation}

In order to map the parameters' space, we need to evaluate the likelihood of a great number of models with respect to the fiducial model and its covariance matrix. For each one of those models, the corresponding shear variance $\left\langle\gamma^{2}\right\rangle_{i}$ must be computed and its likelihood is given by,

$$
-2 \ln L_{i}=\left(\left\langle\gamma^{2}\right\rangle_{i}-\left\langle\gamma^{2}\right\rangle_{f i d}\right)^{T} C^{-1}\left(\left\langle\gamma^{2}\right\rangle_{i}-\left\langle\gamma^{2}\right\rangle_{f i d}\right)
$$

The models where the likelihood is to be computed are not pre-determined on a grid, but are Monte Carlo generated, using the Metropolis-Hastings algorithm (Christensen et al. (2001)), which produces a chain of models. Following this method, a first likelihood is evaluated for a randomly chosen model. Then, a second model is defined at a randomly chosen distance vector from the first one. The method is optimized if there is an approximative knowledge of the covariance matrix in the parameters' space. In that case the orientation of the distance vector may be chosen to follow an eigendirection of that covariance matrix, and its size may be taken to be a function of the corresponding eigenvalue. The crucial step of the algorithm is to compare the likelihood of the two models. If the likelihood of the second is greater than the likelihood of the first times a random number between 0 and 1 (to allow for movement in the "uphill" direction), then the second item on the chain of models will be the second model, i.e., the model is accepted to be part of the chain. If, on the contrary, the second model do not pass the criterium, then it is rejected and the next element of the chain is the first model. The iterative repetition of this procedure produces a chain of models whose density, after convergence, is a good estimator of the probability distribution function in the parameters' space.

We produced 3 types of chains :

- Cosmic shear - Use the shear variance fiducial model as "data". Parameters' space explored in 7 dimensions $\left(\omega_{b}, \omega_{c}, h, n_{s}, \Omega_{v}, \alpha_{s}, \sigma_{8}\right)$. Likelihood computed with the CFHTLS-Wide type covariance matrix, no extra priors imposed.

- CMB - Use the WMAP 1 year data TT and TE power spectra and the CBI mosaic odd binning TT. References to these data are Hinshaw et al. (2003), Kogut et al. (2003) and Pearson et al. (2003). 7 parameters were allowed to vary $\left(\omega_{b}, \omega_{c}, h, n_{s}, \alpha_{s}, A_{s}, \tau\right)$. In the likelihood evaluation we made use of the WMAP likelihood code (Verde et al. (2003)) and imposed flatness.

- Cosmic shear + CMB - Use both sets of data. Parameters explored and priors used are the same as in the CMB case. The chains were produced computing the likelihoods with respect to both experiments.

$\dagger$ http://www.cfht.hawaii.edu/Science/CFHTLS/ 


\section{Results}

We will now extract information, regarding the determination of the cosmological parameters, from the obtained samples of the posterior PDF (the chains).

\subsection{Cosmic shear}

First of all, by computing the standard deviations of the chain models, we obtain the approximative $1 \sigma$ precision level that CFHTLS-Wide can attain. These are shown in Table 2.

\begin{tabular}{cccccccc}
\hline & $\omega_{b}$ & $\omega_{c}$ & $h$ & $n_{s}$ & $\Omega_{v}$ & $\alpha_{s}$ & $\sigma_{8}$ \\
\hline $1 \sigma$ & 0.020 & 0.047 & 0.129 & 0.176 & 0.155 & 0.073 & 0.104 \\
\hline
\end{tabular}

Table 2. Prospective CFHTLS $1 \sigma$ precisions on individual parameters.

The statistical structure of the cosmological parameter space may be further explored by searching for degeneracies between parameters. For this we derive the 7 -dimensional eigenvectors of the parameters' scaled covariance matrix $\left(C_{i j} /<p_{i}><p_{j}>\right.$ ), (shown in Table 3).

\begin{tabular}{lrrrrrrrr}
\hline pc & $1 \sigma$ & $\Omega_{b}$ & $\Omega_{m}$ & $h$ & $n_{s}$ & $\Omega_{v}$ & $\alpha_{s}$ & $\sigma_{8}$ \\
\hline$Y_{1}$ & 0.007 & 0.009 & 0.404 & -0.040 & -0.070 & 0.207 & 0.000 & 0.887 \\
$Y_{2}$ & 0.022 & -0.082 & 0.167 & 0.388 & 0.889 & -0.143 & 0.027 & 0.046 \\
$Y_{3}$ & 0.103 & 0.084 & -0.490 & -0.287 & 0.088 & -0.714 & 0.068 & 0.384 \\
$Y_{4}$ & 0.193 & 0.124 & -0.231 & 0.872 & -0.357 & 0.141 & 0.003 & 0.148 \\
$Y_{5}$ & 0.302 & 0.175 & -0.677 & -0.031 & 0.246 & 0.634 & 0.124 & 0.176 \\
$Y_{6}$ & 0.811 & -0.904 & -0.235 & 0.041 & -0.044 & 0.055 & -0.332 & 0.102 \\
$Y_{7}$ & 1.936 & 0.350 & -0.038 & -0.025 & 0.080 & 0.007 & -0.932 & 0.176 \\
\hline
\end{tabular}

Table 3. Principal components of the statistical distribution of cosmological parameters as probed by cosmic shear.

Consider, for example, the vector $Y_{2}$. From Table 3 we can read its coordinates in the base formed by the 7 cosmological parameters and also, in the second column, its size. This size is the precision with which a new parameter $Y_{2}$, defined as a linear combination of the base parameters, can be determined. It follows $Y_{2}$ is a quantity well determined by cosmic shear and its main contributions come from $h$ and $n_{s}$. On the other hand, there is another $h, n_{s}$ dependent quantity, $Y_{4}$, which is worse determined. Notice the projections of $Y_{2}$ and $Y_{4}$ on the $\left(h, n_{s}\right)$ plane are approximately mutually orthogonal. This shows the existence of a degeneracy in the $\left(h, n_{s}\right)$ plane.

Table 3 shows there are 2 quantities to each cosmic shear is particularly sensitive, $Y_{1}$ and $Y_{2}$. By finding linear combinations of the logarithms of the parameters, we may write the 2 quantities and their corresponding $1 \sigma$ CFHTLS-wide determinations as:

$$
\sigma_{8} \Omega_{m}^{0.52}=0.467 \pm 0.008
$$

and

$$
n_{s}^{0.6} \Omega_{m} h=0.187 \pm 0.037 \text {. }
$$

The first one is the well-known $\sigma_{8}-\Omega_{m}$ "banana shaped" degeneracy and the second one shows a correlation between primordial and current power spectrum slopes. 


\subsection{Cosmic shear $+C M B$}

We turn now to the chains that include CMB information. The parameters' standard deviations obtained with the joint chains are smaller by the factors shown in Table 4, than the standard deviations obtained with the CMB chains.

\begin{tabular}{cccccccc}
\hline & $\omega_{b}$ & $\omega_{c}$ & $h$ & $n_{s}$ & $\alpha_{s}$ & $\sigma_{8}$ & $\Omega_{m}$ \\
\hline gain & 1.4 & 3.6 & 1.9 & 1.7 & 1.7 & 2.5 & 2.8 \\
\hline
\end{tabular}

Table 4. Gain in parameters' precisions when cosmic shear information is included.

The appreciable gain brought by the cosmic shear arises because there is orthogonality between some of cosmic shear degeneracies and CMB degeneracies: both experiments are sensitive to different combinations of the cosmological parameters. In particular, the cosmic shear best constrained directions, $Y_{1}$ and $Y_{2}$, define contours that are orthogonal to their CMB counterparts. This is illustrated in Fig. 1. Notice also the orthogonality in the $n_{s}-\alpha_{s}$ plane, which shows cosmic shear may complement CMB information for the measurement of an eventual primordial power spectrum scale dependence, even though cosmic shear by itself is not particularly sensitive to that parameter $\left(\alpha_{s}\right)$. This complementarity comes essentially from the non-linear small scale information brought by the cosmic shear. Since as $n_{s}$ tilts the spectrum and $\alpha_{s}$ bends it, the response of the spectrum to a change in these parameters will be detected in opposite ways by two experiments that are mainly sensitive to the larger scales of the spectrum (the CMB) or to the smaller scales of the spectrum (the cosmic shear).
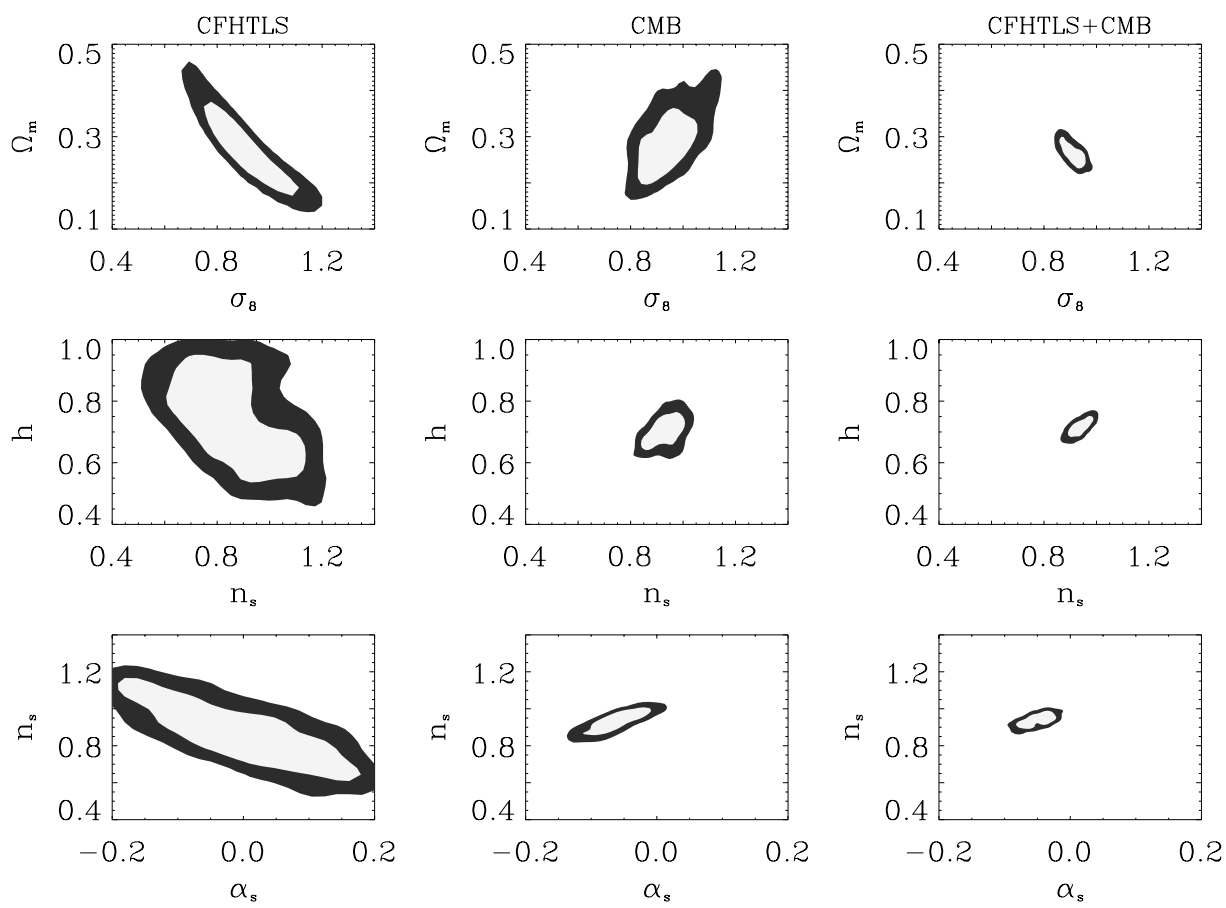

Figure 1. Marginalized 2-dimensional $68 \%$ and $95 \%$ contours from the three samples. These are the most relevant plots to illustrate the large gains on the parameters precisions. 


\section{Conclusion}

We studied the determination of cosmological parameters by a cosmic shear CFHTLSwide type of experiment and found the best constrained parameters to be $\sigma_{8} \Omega_{m}^{0.52}$ and $n_{s}^{0.6} \Omega_{m} h$. We then compared 2-dimensional cosmic shear degeneracies with CMB ones and found some orthogonal cases, namely, $\left(\sigma_{8}, \Omega_{m}\right),\left(n_{s}, h\right)$ and $\left(n_{s}, \alpha_{s}\right)$. This led us to predict a gain in the parameters' precisions when both experiments are combined, of the order 1.5-2.5 for several parameters, including $\alpha_{s}$, which shows second generation cosmic shear surveys may probe non-standard cosmologies.

The exact factors of gain attained by the CFHTLS-wide are subject to changes depending on how well systematics (which were not included in this study) are handled. In particular, an efficient PSF correction and complete removal of systematics residuals that show up as B-mode contamination is crucial.

Besides the control of the systematics, other assumptions were made in this study that we which to point out. Firstly, we assumed all CFHTLS-wide survey area is usable, whereas the masking process may reduce by about $20 \%$ the total sky coverage of deep surveys. Secondly, we assumed that the source redshift distribution was perfectly known. In reality, there is an extra source of error coming from the marginalization over the real sources redshift distribution. The same happens with the marginalization over other cosmological parameters not taken into account in this study, such as the equation of state of dark energy or the neutrinos density. Finally, the precision of the non-linear mapping used in our calculations into deep non-linear regime is another source of uncertainty.

The uncertainties arising from these 3 issues would degrade the CFHTLS-wide determinations by a factor of 1.9 and the factors of gain of the joint analysis by a factor of 1.3 (for details see Tereno et al. (2004)).

But most importantly, these issues do not change the degeneracies found, thus the cosmic shear potential to complement CMB remains unaltered.

\section{Acknowledgements}

The author thanks the organizers (and some of the participants) for this interesting meeting and acknowledges financial support from a Fundação para a Ciência e a Tecnologia (FCT) scholarship and a Programme National de Cosmologie (PNC) displacement grant.

\section{References}

Bacon, D., Taylor, A., Brown, M. et al. 2004, astro-ph/0403384

Bartelmann, M. \& Schneider, P. 2001, Phys. Rep., 340, 291

Benabed, K. \& van Waerbeke, L. 2003, astro-ph/0306033

Christensen, N., Meyer, R., Knox, L. \& Luey, B. 2001, Class. Quant. Grav., 18, 2677

Contaldi, C., Hoekstra, H. \& Lewis, A. 2003, Phys. Rev. Lett., 90, 1303

Hinshaw, G., Spergel, D.N., Verde, L. et al. 2003, ApJS, 148, 135

Ishak, M., Hirata, C., McDonald, P. \& Seljak, U. 2004, Phys. Rev. D, 69, 083514

Kogut, A., Spergel, D.N., Barnes, C. et al. 2003, ApJS, 148, 161

Lewis, A., Challinor, A. \& Lasenby, A. 2000, ApJ, 538, 473

Pearson, T. J., Mason, B., Readhead, A. et al. 2003, ApJ, 591, 556

Schneider, P., van Waerbeke, L., Kilbinger, M. \& Mellier, Y. 2002, A\& A, 396, 1

Smith, R., Peacock, J., Jenkins, A. et al. 2003, MNRAS, 341, 1311

Takada, M. \& Jain, B. 2004, MNRAS, 348, 897

Tereno, I., Doré, O., van Waerbeke, L. \& Mellier, Y. 2004, astro-ph/0404317

Van Waerbeke, L. \& Mellier, Y. 2003, astro-ph/0305089

Verde, L., Peiris, H.V., Spergel, D.N. et al. 2003, ApJS, 148, 195 